\title{
Magnetic Field Effect on Sound Propagation in Antiferromagnets
}

\author{
A. PaWlak and B. Fechner \\ Faculty of Physics, Adam Mickiewicz University \\ Umultowska 85, 61-614 Poznań, Poland
}

\begin{abstract}
On the basis of the theory of phase transitions, a model describing anomalies of sound attenuation coefficient close to the antiferromagnet-paramagnet phase transition in magnetic field was developed. The scaling behaviour of the sound velocity and attenuation coefficient was determined. The physical origin of the two-peak structure in the field dependent ultrasound attenuation observed in a number of antiferromagnets was identified. The theoretical results were compared with experimental results obtained for terbium.
\end{abstract}

PACS numbers: 05.70.Jk, 62.65.+k

\section{Introduction}

The interest in antiferromagnetic spin systems has grown considerably during the last decade. Many experimental and theoretical studies have been made on anomalous ultrasonic behaviour near the transition temperature $[1,2]$. Much of the effort has been devoted to understanding of the effect of the external field on the critical sound propagation [3-5]. In antiferromagnets the weak external magnetic field usually does not destroy the continuous phase transition both for the magnetic field lying parallel to the antiferromagnetic order parameter as well as for that perpendicular to the order parameter. High magnetic fields may change the phase transition into the first order one as for example in metamagnets [6]. In low magnetic fields, the Néel temperature changes according to the relation: $T_{\mathrm{N}}(H)=T_{\mathrm{N}}(0)-a H^{\zeta}$, where the exponent $\zeta$ is equal to 2 in the mean field approximation. The situation is very similar to that in the ferromagnet in a perpendicular field where the field causes a shift in the transition temperature and does not destroy the continuous transition. As a consequence of a shift of the Néel temperature the peak in the sound attenuation and the minimum in the sound velocity are also shifted towards lower temperatures. The scaling relations for the sound attenuation coefficient $\alpha(T, \omega, H)$ and the sound velocity $c(T, \omega, H)$ take the following forms:

$$
\begin{aligned}
& \alpha(T, \omega, H)=\omega^{2} t(H)^{-\rho_{\mathrm{s}}} f\left(\omega t(H)^{-z \nu}\right), \\
& c^{2}(T, \omega, H)-c_{0 \pm}^{2}= \pm t(H)^{ \pm \alpha} g\left(\omega t(H)^{-z \nu}\right),
\end{aligned}
$$

where $t(H)=\frac{T-T_{\mathrm{N}}(H)}{T_{\mathrm{N}}(H)}$ is the reduced temperature measuring the distance to the critical point, $\rho_{\mathrm{s}}$ is the sound attenuation critical exponent and $f, g$ are scaling functions [7-9] (which can be different above and below the Néel temperature); $c_{0 \pm}$ are background sound velocities. Generally, the sound attenuation exponent takes differ- ent values for two classes of magnetic materials. It is $2 \alpha$ for the magnets being also insulators ( $\alpha$ is the specific heat critical exponent) and $\rho_{\mathrm{s}}=z \nu \pm \alpha$ in magnetic metals, where $z$ is the dynamic critical exponent and $\nu$ the correlation length exponent. The plus sign refers to low-frequency regime and minus to the high-frequency regime in metals [7]. In Eqs. (1) and (2) it is understood that the system is far from a multicritical point such as a tricritical, bicritical or tetracritical one which is expected for sufficiently high magnetic fields $[10,11]$ in many antiferromagnets.

\section{Position of the sound attenuation peak}

Essentially the same expressions apply to ferromagnets in perpendicular field. When the magnetic field is applied along the hard axis, the transverse field does not destroy the continuous transition and only shifts the Curie temperature so that the peak in the sound attenuation coefficient is moved towards lower temperatures as a result of decreasing Curie temperature. In a ferromagnet in transverse magnetic field, the magnetic field $H$ should be replaced by the perpendicular field $H_{\perp}$ and the Néel temperature by the Curie temperature $T_{\mathrm{C}}\left(H_{\perp}\right)$.

The narrow peak in the sound attenuation is also shifted in both cases. The temperature at which the sound attenuation coefficient has its maximum is described by the equation

$$
T_{\max }(H)-T_{\mathrm{N}}(0)=-a \omega^{z v}-b H^{2}-c H^{4} .
$$

Expression (3) applies also to the ferromagnets in the perpendicular field as is illustrated for $\mathrm{MnP}$ in Fig. 1, where $a, b, c$ are some constants and $\omega$ is the ultrasound frequency. The first term in Eq. (3) describes the zero-field shift of the attenuation peak with respect to the critical temperature. It originates from the relaxational 
or the Landau-Khalatnikov contribution to the sound attenuation $[13,14]$ that is the only one which contributes to the sound damping in the mean-field theory. In the Landau-Khalatnikov mechanism the sound attenuation maximum as well as the sound velocity minimum occur slightly below the transition temperature, i.e. at the temperature at which the reduced frequency (in the ordered phase) $y_{-} \approx 1$. The other two terms in Eq. (3) come from the shift of the critical temperature in the mean-field approximation (the term $v M^{6}$ is added to the Landau free energy).

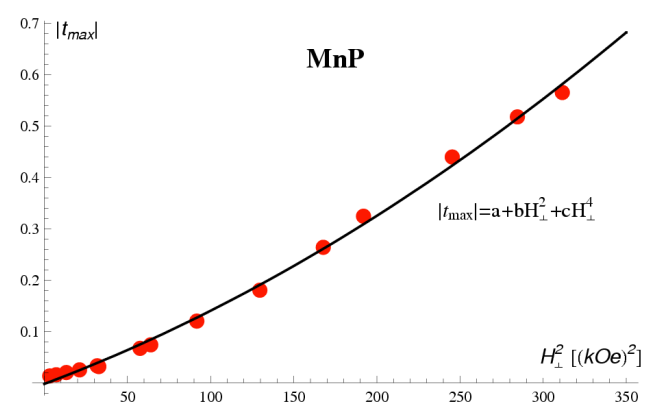

Fig. 1. The plot of $\left|t_{\max }\right|$ in $\mathrm{MnP}$ as the function of $H_{\perp}^{2}$. The parabola corresponds to Eq. (3). The ultrasonic data for $\mathrm{MnP}$ are taken from [12].

\section{Additional broad maximum in the sound attenuation coefficient}

In some antiferromagnets (but not in all) as for example in $\mathrm{Tb}$ [15] or MnSi [16] the attenuation shows a broad peak above the Néel point. The second broad maximum in the sound attenuation coefficient has been also found in the paramagnetic phase of $\mathrm{MnP}$ [12] when a perpendicular field was applied. The effect of the magnetic field on the critical sound damping was investigated by Tachiki and Maekawa [17] who recognised that the additional broad peak above the critical temperature comes from the uniform susceptibility. Its contribution to the sound attenuation is given by

$$
\Delta \alpha \propto \omega M^{2} \operatorname{Im} \chi(\omega, T, H),
$$

where $M$ is the induced magnetic polarisation and $\chi(\omega, T, H)$ is the dynamic susceptibility connected with uniform $(q=0)$ fluctuations. In the Van Hove approximation $\chi(\omega, T, H)=\frac{1}{-\mathrm{i} \omega / \Gamma+\chi(T, H)^{-1}}$ it is determined by the static susceptibility $\chi(T, H)$, where $\Gamma$ is the spin relaxation rate. The crucial point is the field and temperature dependence of the static susceptibility. The general phenomenological model of interacting staggered $N(x)$ and uniform $M(x)$ magnetic order parameters is defined by the following Ginzburg-Landau functional:

$$
\begin{aligned}
F= & \frac{1}{2} \int \mathrm{d}^{d} x\left[r_{1} N^{2}+(\nabla N)^{2}+r_{2} M^{2}+(\nabla M)^{2}\right. \\
& \left.+u_{11} N^{4}+u_{12} N^{2} M^{2}+u_{22} M^{4}-H M\right],
\end{aligned}
$$

where $r_{1} \propto T-T_{\mathrm{N}}^{0}$ and $r_{2} \propto T-T_{\mathrm{C}}^{0}$ with $T_{\mathrm{N}}^{0}$ and $T_{\mathrm{C}}^{0}$ being the bare Néel and Curie temperature, respectively. In antiferromagnets it is assumed that $T_{\mathrm{N}}^{0}-T_{\mathrm{C}}^{0}>0$. The static susceptibility of the paramagnetic phase $(N=0)$ as a function of temperature and magnetic field is shown in Fig. 2 for three different Curie temperatures. It is

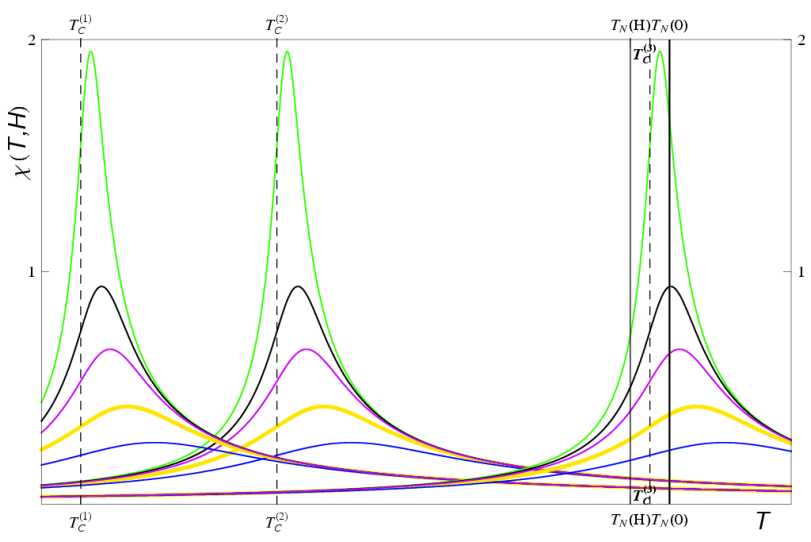

Fig. 2. The susceptibility of the paramagnetic phase as a function of the temperature and magnetic field. The height of the peak decreases with increasing magnetic field. Three different Curie temperatures are considered in order to show that only for $T_{\mathrm{C}} \approx T_{\mathrm{N}}$ the susceptibility peak appears at temperatures higher than $T_{\mathrm{N}}(H)$.

easy to see that only for $T_{\mathrm{C}} \approx T_{\mathrm{N}}$ the susceptibility peak appears at temperatures higher than $T_{\mathrm{N}}(H)$. Thus the Curie temperature should not be much lower than the (zero-field) Néel temperature. As the magnetic field increases the Néel temperature decreases and the temperature $T_{\max }$ at which the uniform susceptibility peak occurs increases. Below the Néel temperature $T_{\mathrm{N}}(H)$, due to the interaction with the staggered magnetisation different expression for the susceptibility applies and the peaks on the left side of $T_{\mathrm{N}}(H)$ are not observed in the experiment. Such a favourable situation of very close magnetic transitions appears in terbium where $T_{\mathrm{N}}-T_{\mathrm{C}} \approx 10 \mathrm{~K}$ $\left(T_{\mathrm{N}} \approx 228.5 \mathrm{~K}\right)$ and second broad peak in the sound attenuation was observed [12]. For a high magnetic field the location of this peak is given by

$$
T_{\max }-T_{\mathrm{N}} \propto H^{\frac{1+z \nu}{\Delta}},
$$

which in the mean-field approximation (MFA) gives $t_{\max }^{\mathrm{P}} \propto H^{\frac{4}{3}}$. In Fig. 3 the plot of $t_{\max }^{\mathrm{P}}$ vs. $H$ for $\mathrm{Tb}$ is given. Index "P" refers to the broad maximum in the paramagnetic phase. The height of this maximum is given by $\alpha_{\max }^{\mathrm{P}} \propto H^{-z \nu / \Delta}$ (for high magnetic fields) which is illustrated in Fig. 4. Closeness of the Curie and Néel temperatures is not a sufficient condition for the occurrence of the second peak in the ultrasonic attenuation. Some relations between the coefficients $u_{i j}$ in Eq. (5) must also be satisfied in order for the transition to remain continuous with increasing magnetic field. For example in the metamagnets we usually expect $u_{11}=u_{22}=u_{12} / 6$ [11] and for very close $T_{\mathrm{C}}$ and $T_{\mathrm{N}}$ the transition becomes first order. On the other hand, for transverse ferromagnets $u_{11}=u_{22}=u_{12} / 2$ [18] the phase transition remains 


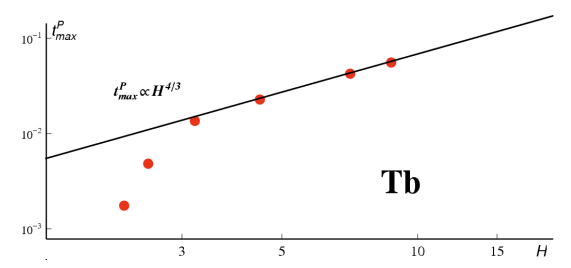

Fig. 3. The reduced temperature $t_{\max }=\frac{T_{\max }^{\mathrm{P}}-T_{\mathrm{N}}(0)}{T_{\mathrm{N}}(0)}$ as a function of the magnetic field. The ultrasonic data for $\mathrm{Tb}$ are taken from [15].

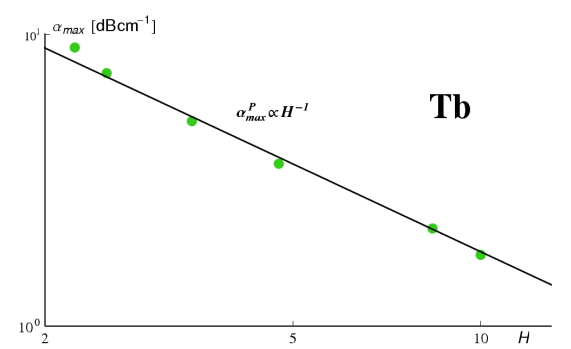

Fig. 4. The height of the broad ultrasonic attenuation peak in the paramagnetic phase as a function of the magnetic field. The data are from [15].

continuous for $T_{\mathrm{C} 2}$ approaching $T_{\mathrm{C} 1}$, where $T_{\mathrm{C} 1}$ is the easy axis Curie temperature and $T_{\mathrm{C} 2}$ is the corresponding temperature for the perpendicular axis.

\section{References}

[1] A. Pawlak, Phys. Status Solidi C 3, 204 (2006).
[2] A. Pawlak, B. Fechner, Phys. Status Solidi C 3, 208 (2006).

[3] G. Gulpinar, Phys. Lett. A 372, 98 (2008).

[4] R.K Zheng, C.F. Zhu, X.G. Li, Phys. Status Solidi A 184, 251 (2001).

[5] O. Chiatti, A. Sytcheva, J. Wosnitza, S. Zherlitsyn, A.A. Zvyagin, V.S. Zapf, M. Jaime, A. Paduan-Filho, Phys. Rev. B 78, 094406 (2008).

[6] J.M. Kincaid, E.G.D. Cohen, Phys. Rep. 22, 57 (1975).

[7] A. Pawlak, Eur. Phys. J. B 4, 179 (1998).

[8] A. Pawlak, Sound Propagation near the Magnetic Phase Transition, Wydawnictwo Naukowe UAM, Poznań 2004 (in Polish).

[9] A. Pawlak, Phys. Status Solidi B 236, 474 (2003).

[10] J.M. Kosterlitz, D.R. Nelson, M.E. Fisher, Phys. Rev. B 13, 412 (1976).

[11] D.R. Nelson, M.E. Fisher, Phys. Rev. B 11, 1030 (1976).

[12] T. Komatsubara, A. Ishizaki, S. Kusaka, E. Hirahara, Solid State Commun. 14, 741 (1974).

[13] L.D. Landau, I.M. Khalatnikov, Dokl. Akad. Nauk SSSR 96, 469 (1954); reprinted in Collected Papers of L.D. Landau, Ed. D. ter Haar, Pergamon, London 1965.

[14] B. Lüthi, Physical Acoustics in the Solid State, Springer, Berlin 2005.

[15] S. Maekawa, R.A. Treder, M. Tachiki, M.C. Lee, M. Levy, Phys. Rev. B 13, 1284 (1976).

[16] S. Kusaka, K. Yamamoto, T. Komatsubara, Y. Ishikawa, Solid State Commun. 20, 925 (1976).

[17] M. Tachiki, S. Maekawa, Prog. Theor. Phys. 51, 1 (1974).

[18] J. Sznajd, Acta Phys. Pol. A 40, 637 (1971). 